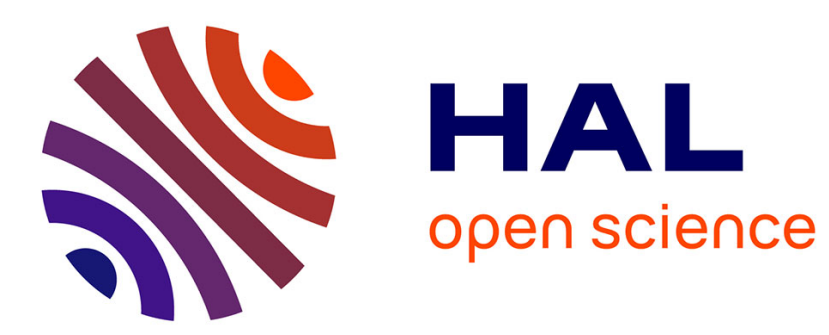

\title{
Brillouin instabilities in continuously pumped high power fiber lasers
}

Djouher Mallek, Abdelhamid Kellou, Hervé Leblond, François Sanchez

\section{To cite this version:}

Djouher Mallek, Abdelhamid Kellou, Hervé Leblond, François Sanchez. Brillouin instabilities in continuously pumped high power fiber lasers. Journal of Nonlinear Optical Physics and Materials, 2009, 18 (01), pp.111-120. 10.1142/S0218863509004476 . hal-03423778

\section{HAL Id: hal-03423778 \\ https://univ-angers.hal.science/hal-03423778}

Submitted on 10 Nov 2021

HAL is a multi-disciplinary open access archive for the deposit and dissemination of scientific research documents, whether they are published or not. The documents may come from teaching and research institutions in France or abroad, or from public or private research centers.
L'archive ouverte pluridisciplinaire HAL, est destinée au dépôt et à la diffusion de documents scientifiques de niveau recherche, publiés ou non, émanant des établissements d'enseignement et de recherche français ou étrangers, des laboratoires publics ou privés. 


\title{
BRILLOUIN INSTABILITIES IN CONTINUOUSLY PUMPED HIGH POWER FIBER LASERS
}

\author{
DJOUHER MALLEK ${ }^{*, \dagger}$, ABDELAMID KELLOU*, \\ HERVÉ LEBLOND ${ }^{\dagger}$ and FRANÇOIS SANCHEZ ${ }^{\dagger, \ddagger}$ \\ *Laboratoire d'Electronique Quantique, Faculté de Physique, \\ Université des Sciences et de la Technologie Houari Boumediene, \\ B. P. 32 El-Alia Bab-Ezzouar 16111, Alger, Algeria \\ ${ }^{\dagger}$ Laboratoire POMA FRE CNRS 2988, Université d'Angers, \\ 2 Bd Lavoisier, 49000 Angers, France \\ ${ }_{\ddagger}^{\ddagger}$ rancois.sanchez@univ-angers.fr
}

Received 26 February 2009

\begin{abstract}
The effect of Brillouin backscattering on the stability of a high power continuously pumped fiber laser is theoretically analyzed in the general framework of two-coupled modes laser model. It is demonstrated that, depending on the cavity losses, different type of instabilities can arise. Low loss cavity favours stable continuous regime in a large range of pumping rates while high loss configuration permits the emergence of different self-pulsing instabilities.
\end{abstract}

Keywords: Fiber laser; Brillouin effect; instabilities; theoretical model.

\section{Introduction}

Brillouin backscattering and its related instabilities in single-mode passive fibers have a long history. ${ }^{1}$ The physical principle is the parametric coupling between an optical and an acoustical wave in a long single-mode fiber. ${ }^{2}$ Electrostrictive effect induces distributed moving index grating in which the pump beam is reflected thus generating the backscattered Stokes wave which is frequency down-shifted. The simplest configuration involves only a long fiber without any feedback and a forward pump beam. Chaotic behaviour is observed in this case even close to the Brillouin threshold. ${ }^{1}$ External feedback provided by the fiber ends leads to greater rich dynamical behaviours such as limit cycle and quasiperiodicity. ${ }^{1,3}$ Theoretical modelling allowed to identify the nonlinear refraction and its interplay with the Brillouin coupling as responsible for the dynamical behaviours. Indeed, if the optical Kerr effect is omitted, no instability is observed. ${ }^{4}$ Thus the dynamics was explained in a purely deterministic way. ${ }^{5}$ On the other hand, an alternative approach to explain the chaotic behaviour without external feedback was related to the stochastic nature of the spontaneous scattering. ${ }^{6,7}$ Further studies demonstrated that external feedback suppresses the stochasticity of the Brillouin interaction process leading to a purely 
deterministic behaviour resulting from the interplay between nonlinear refraction and Brillouin amplification. ${ }^{8,9}$ Brillouin fiber ring lasers have been also deeply investigated both experimentally and theoretically. Here again, different dynamic instabilities have been obtained depending of the fiber length. ${ }^{10-12}$ Although several mechanisms or cavity characteristics are involved in the destabilization process, the nonlinear refraction is a necessary condition. ${ }^{12}$

With the emergence of rare-earth doped fibers, and especially double-clad fibers, there is a renewed interest in Brillouin effect. First of all, the amplification of a continuous signal in a rare-earth doped fiber amplifier can generate high enough intensities to excite Brillouin effect and then to create a backscattered Stokes wave. Such back-reflection is detrimental for amplifier applications and consequently it has been studied theoretically and experimentally. The main objective was initially to calculate the Brillouin threshold in an amplifying medium. ${ }^{13-15}$ Time-dependent analysis in a double-clad fiber amplifier has been first done in Ref. 16 where the authors investigate the effect of multiple Brillouin scattering on microsecond-pulse amplification. There are few reported results concerning Brillouin effect in a resonant fiber laser. First experimental results concerned a double-clad ytterbiumdoped fiber laser where Brillouin scattering was suspected to be responsible for the unstable behaviour of the laser. ${ }^{17}$ Indeed, the laser was stabilized in a unidirectional ring cavity where backscattering is suppressed. Clear experimental evidence of multiple stokes waves and their destabilizing effect has been reported. ${ }^{18}$ The cavity losses have been identified as an important parameter directly linked to the laser behaviour. ${ }^{19}$ Indeed, the laser is $\mathrm{cw}$ in a low-loss configuration while different self-pulsing instabilities occur in high-loss cavity. Very few results have been reported from the theoretical point of view. We can cite a work which concerns the modelling of self-Q-switching of a fiber laser cavity with a Brillouin scattering ring mirror. ${ }^{20}$ More recently, the influence of Brillouin scattering in a fiber laser actively Q-switch has been theoretically modelled. ${ }^{21}$ In this paper we report, for the first time to the best of our knowledge, theoretical results for the low frequency self-pulsing instability resulting from Brillouin backscattering. In contrast with, ${ }^{21}$ we consider an autonomous system with no external or internal modulation. We give in this paper the most important results. Further developments will be let future papers. Our main objective is to present a model allowing to explain the low frequency instability arising in a fiber lasers as a consequence of Brillouin effect.

\section{The Model}

We consider the Fabry-Perot fiber laser cavity schematically depicted in Fig. 1. We assume that the fiber supports a forward and a backward laser waves which are modelled through their intensities. For simplicity we assume that there is only the first order stokes wave with its forward and backward components. Indeed, experimental results reported in Ref. 18 showed that Brillouin effect destabilizes the laser but no clear evidence was done concerning a possible correlation between the number of Stokes orders and the stability of the laser. In a first approach we neglect 


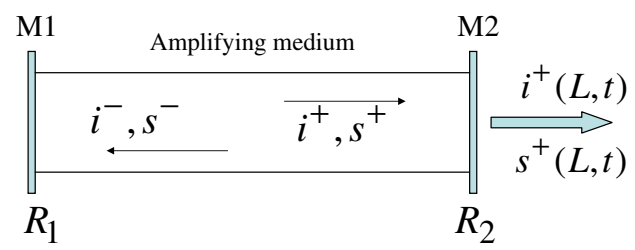

Fig. 1. Laser configuration and notations used in this paper.

the equation for the acoustic wave and the optical Kerr effect. Of course these two aspects can be of importance in special situations but, as it will be demonstrated, they are not key elements for the description of Brillouin induced instabilities in a standing-wave fiber laser. The neglecting of the acoustic wave means that we assume an instantaneous Brillouin gain, while in fact the phonon lifetime is about 15 ns. The consequence of our approach is that the model is intrinsically limited to a description of phenomena in a time scale well above the phonon lifetime. In spite of such limitation, it is sufficient for the modelling of both the low frequency self-pulsing occurring in the microsecond range and the fast pulsing occurring in the round-trip time scale, about $100 \mathrm{~ns}$.

The different waves are described in terms of intensities which are subjected to the classical boundary conditions on the mirrors. The forward (backward) component of the laser wave is coupled to the backward (forward) component of the stokes wave as a result of the Brillouin coupling. In addition, all waves fall below the gain curve and consequently are coupled through the gain medium. At this stage we have two possibilities. The first one is to assume that all the fields are amplified through stimulated emission associated to a unique population inversion. This approach, used in Ref. 21, does not seem satisfactory because the spatial hole burning in standing-wave lasers yields naturally to the existence of different classes of dipoles. ${ }^{22}$ The second approach, that we decided to use, is a consequence of the spatial hole burning and consists in considering two classes of atoms, each associated to one wavelength. ${ }^{22}$ Such approach has been used successfully for the description of the dynamics of dual-wavelength erbium-doped fiber lasers. ${ }^{23}$ Of course, crosssaturation occurs between the laser and the Stokes waves. In its final form, the model involves 2 material equations (population inversions) and 4 field equations (forward and backward components of both laser and stokes waves). The normalized equations write as

$$
\begin{aligned}
\frac{\partial d_{1}}{\partial t} & =p-a_{1}\left(1+d_{1}\right)-2 d_{1}\left[\left(i^{+}+i^{-}\right)+\beta\left(s^{+}+s^{-}\right)\right] \\
\frac{\partial d_{2}}{\partial t} & =\gamma p-a_{1}\left(1+d_{2}\right)-2 d_{2}\left[\left(s^{+}+s^{-}\right)+\beta\left(i^{+}+i^{-}\right)\right] \\
\frac{\partial i^{ \pm}}{\partial t} \pm \frac{\partial i^{ \pm}}{\partial \zeta} & =-G i^{ \pm} s^{\mp}-k i^{ \pm}+A\left(d_{1}+\beta d_{2}\right) i^{ \pm} \\
\frac{\partial s^{ \pm}}{\partial t} \pm \frac{\partial s^{ \pm}}{\partial \zeta} & =+G i^{\mp} s^{ \pm}-k s^{ \pm}+A\left(d_{2}+\beta d_{1}\right) s^{ \pm}
\end{aligned}
$$


with the reflection conditions at the boundaries

$$
\begin{aligned}
& i^{+}(0, t)=R_{1} \times i^{-}(0, t), \quad s^{+}(0, t)=R_{1} \times s^{-}(0, t), \\
& i^{-}(\ell, t)=R_{2} \times i^{+}(\ell, t), \quad s^{-}(\ell, t)=R_{2} \times s^{+}(\ell, t) .
\end{aligned}
$$

The $d_{n}$ 's are the population inversions normalized versus the concentration of active ions $\left(N_{0}=10^{24} \mathrm{~m}^{-3}\right) . i$ and $s$ are the normalized laser and stokes intensities, respectively and the superscript plus and minus stand for forward and backward components, respectively. The time $t$ is normalized versus the photon transit time along the fiber $\left(T_{r}=48 \mathrm{~ns}\right)$ and the longitudinal coordinate $\zeta=z / L$ is normalized versus the fiber length $(L=10 \mathrm{~m})$ which is assumed to coincide with the total cavity length, so that the normalized cavity length is $\ell=1$. The other parameters are $a_{1}=T_{r} / \tau, A=\sigma N_{0} L, G=g_{B} L I_{0}, I_{0}=\hbar \omega /\left(\sigma T_{r}\right)$ and $k=\alpha L . \tau=800 \mu s$ is the population inversion lifetime, $\sigma=32 \times 10^{-25} \mathrm{~m}^{2}$ the emission cross-section, $g_{B}$ the Brillouin gain and $\alpha=0.0458 \mathrm{~m}^{-1}$ the absorption coefficient. $\omega=2 \pi c / \lambda$ is the optical pulsation with $\lambda=1.08 \mu \mathrm{m}$. $p$ is the pumping parameter and is assumed to be uniform along the fiber. $\gamma$ represents a dichroism in the pumping process in order to take into account some anisotropy of the laser. ${ }^{24} \beta$ is the crosssaturation parameter and physically represents the strength of the coupling between the laser field and the stokes through the amplifying medium. ${ }^{22-24} R_{1}$ and $R_{2}$ are the reflection coefficients of the mirrors.

Before we proceed, it is worth discussing the value of the Brillouin gain because it has a significant influence on the numerical results. In addition, depending on the exact experimental conditions, $g_{B}$ can take very different values. The commonly used Brillouin gain in silicate fibers is $g_{B}^{m}=5 \times 10^{-11} \mathrm{~m} / \mathrm{W}$ and corresponds to its maximum value at the centre of the Brillouin line. Let us now specify the exact operating conditions of the laser under investigation. First of all, the ratio of the spectral Brillouin gain linewidth $\left(\Delta \nu_{B}\right)$ and the spectral laser linewidth $\left(\Delta \nu_{P}\right)$ plays an important role. We consider a situation where $\Delta \nu_{B}=10 \mathrm{MHz}^{25}$ and $\Delta \nu_{P}=$ $100 \mathrm{MHz}$. Thus we consider a configuration where $\Delta \nu_{P} \gg \Delta \nu_{B}$. As a consequence, the Brillouin gain considerably varies depending on the respective values of the coherence length of the laser $\left(L_{\mathrm{coh}}=c /\left(n \Delta \nu_{P}\right)\right.$ where $c$ is the velocity of light and $n \approx 1.45$ the refractive index) and the Brillouin interaction length $\left(L_{\mathrm{int}}=1 /\left(g_{B}^{m} I\right)\right.$ where $I$ is the laser intensity). The coherence length can be evaluated with the spectral laser linewidth considered above $L_{\text {coh }} \approx 2 \mathrm{~m}$. To determine the interaction length, we consider a wave with a total power of about $1 \mathrm{~W}$ and an effective mode area for the fundamental mode of about $25 \mu \mathrm{m}^{2}$. We get $L_{\text {int }} \approx 0.5 \mathrm{~m}$. Therefore we are in the case where $L_{\text {coh }} \gg L_{\text {int }}$. We finally notice that the free spectral range of the cavity $\left(\Delta \nu_{F S R} \approx 11 \mathrm{MHz}\right)$ is greater than the Brillouin gain linewidth $\left(\Delta \nu_{B}=10 \mathrm{MHz}\right)$. Under all these circumstances, the Brillouin gain to be considered is $g_{B}=\left(\Delta \nu_{B} / \Delta \nu_{P}\right) g_{B}^{m}=g_{B}^{m} / 10$. In conclusion, with the set of parameters used for the numerical simulations we will consider a Brillouin gain which is 10 times lower than its maximum value. 


\section{Numerical Simulations}

System (1-2) is solved numerically using a 4th order Runge-Kutta algorithm. The $\zeta$-derivatives are computed by means of finite differences and care must be taken to the reflection conditions (2). In the following we are interested in the temporal evolution of the output intensity of the laser $\left(i^{+}(\ell, t)\right)$ and stokes $\left(s^{+}(\ell, t)\right)$ waves and also on the total output intensity defined as $i_{\text {tot }}=i^{+}(\ell, t)+s^{+}(\ell, t)$. For the numerical simulations we consider the practical case of the Yb-doped double-clad fiber laser. All the parameters are known except the values of $G, \gamma$ and $\beta$ which are chosen in order to obtain a good qualitative agreement with the experimental observations reported in Refs. 17-19, we will take the normalized Brillouin gain $G=60$ (in agreement with the discussion given in the previous paragraph), the dichroism parameter $\gamma=0.7$ and the cross-saturation parameter $\beta=0.05$. The cavity losses, through the choice of the reflection coefficients of the mirrors, together with the pumping ratio $r$ (defined as the ratio of the pumping parameter to its laser threshold value $r=p / p_{\text {th }}$ ) have a strong influence on the dynamical behaviour and will be varied hereafter. The laser threshold can be directly calculated from system (1) evaluated at threshold and then by considering the fact that the gain compensates the losses over one round-trip of the cavity. It takes the form:

$$
p_{\text {th }}=\frac{a_{1}}{1+\beta \gamma}\left[1+\beta+\frac{1}{A}\left(k+\frac{1}{2 \ell} \operatorname{Ln} \frac{1}{R_{1} R_{2}}\right)\right]
$$

We first consider the case of a high-losses cavity (or bad cavity configuration) for which the reflection coefficients of the mirrors are $R_{1}=1$ and $R_{2}=0.16$. Results of the numerical integration are given in Fig. 2. Figure 2(a) has been obtained for a pumping ratio $r=6.33$ and clearly demonstrates a regular self-pulsing instability in the microsecond range. While the pumping ratio is increased, this regular self-pulsing progressively becomes irregular with the emergence of additional fast modulation as shown in Figs. 2(b) and 2(c). Further work is needed to fully characterize the instability but the results are yet enough important at least for two reasons. First, they are in very good qualitative agreement with the experimental data reported in Refs. 17,18 and, they demonstrate for the first time that Brillouin effect can be responsible for a low-frequency self-pulsing instability in a fiber laser without any saturable absorber. We can note that the lower period in the time evolution exactly matches the round-trip cavity time (here $96 \mathrm{~ns}$ ) as in the experiment. In order to be fully convinced that these instabilities are due to the Brillouin effect, we have checked that when $G=0$, no instability occurs and the laser is continuous. This is clearly demonstrated in Fig. 3 which gives the temporal evolution of the output intensity for the same set of parameters than that of Fig. 2 but with no Brillouin coupling.

Let us now consider the low-loss cavity case (good cavity configuration) for which the reflection coefficients of the mirrors are $R_{1}=1$ and $R_{2}=0.9$. In this case the laser is continuous as shown in Fig. 4 which gives the transient evolution towards a stable steady-state. The laser remains stable over a large range of pumping ratio. 


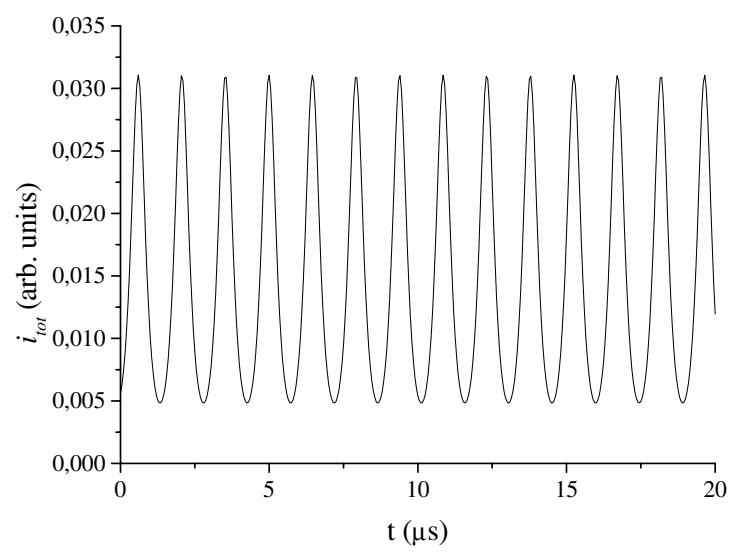

(a)

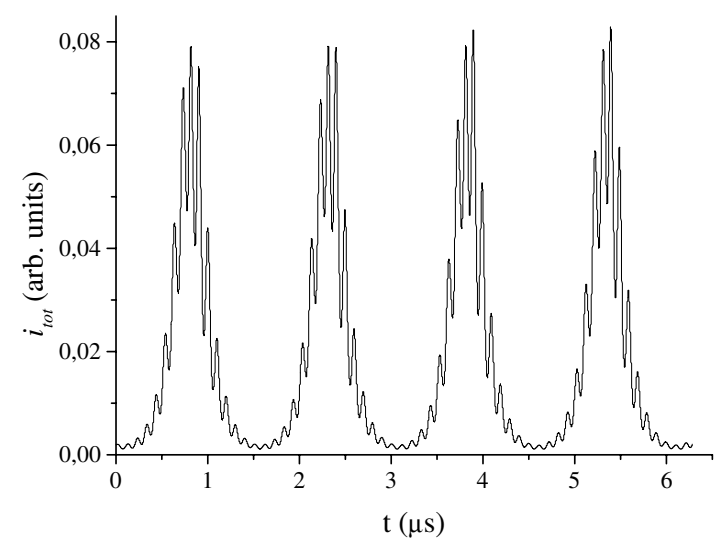

(b)

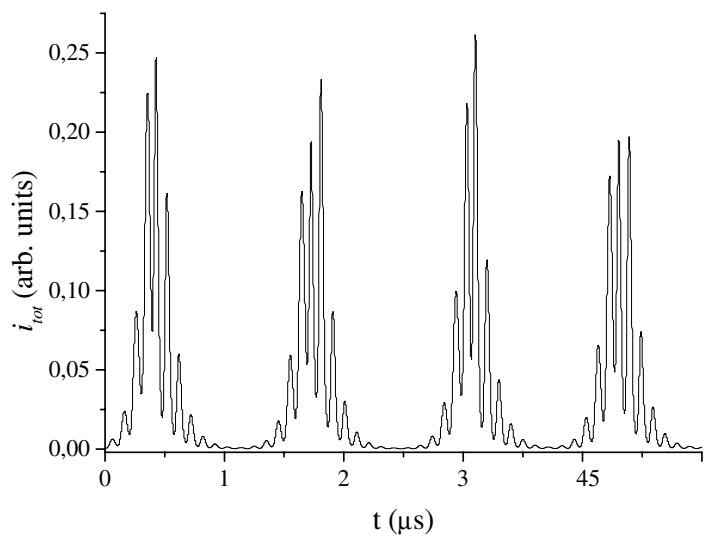

(c)

Fig. 2. Temporal evolution of the output laser intensity in a high-loss cavity with Brillouin coupling. The pumping ratio is (a) $r=6.33$, (b) $r=7.79$ and (c) $r=11.68$. 


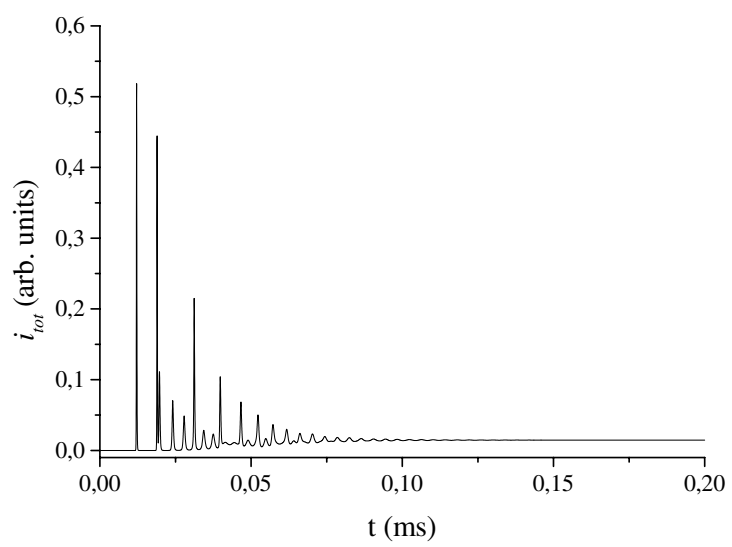

(a)

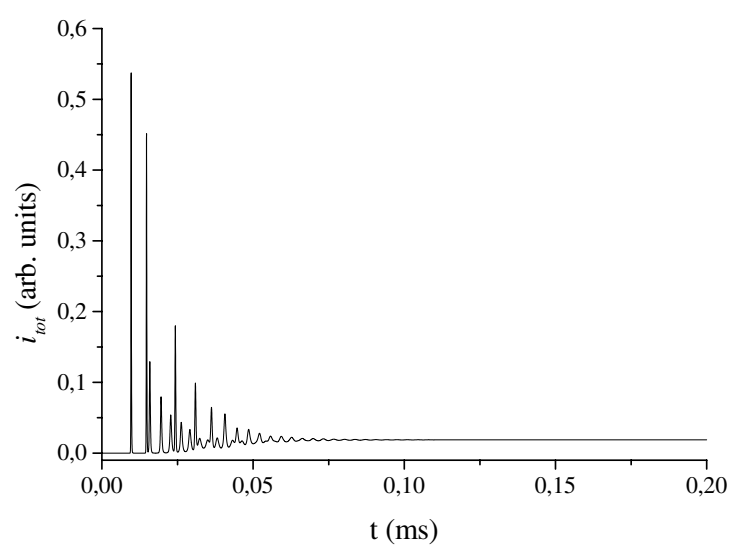

(b)

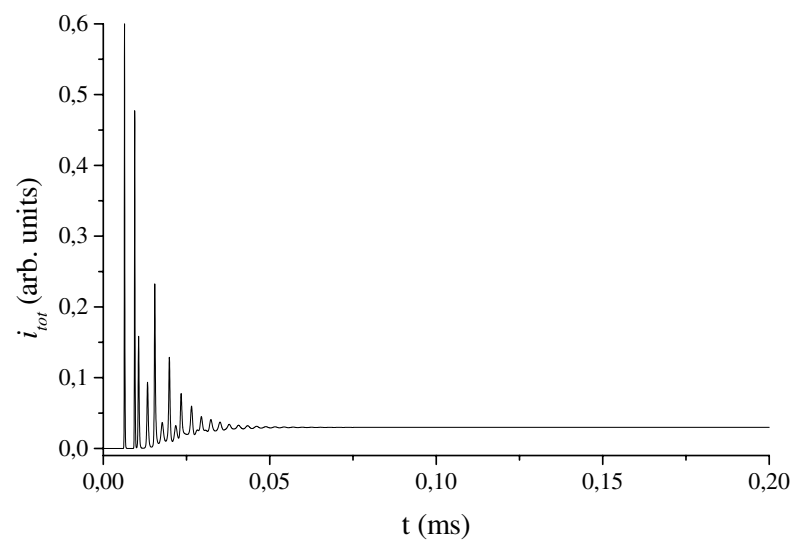

(c)

Fig. 3. Temporal evolution of the output laser intensity in a high-loss cavity without Brillouin coupling. The pumping ratio is (a) $r=6.33$, (b) $r=7.79$ and (c) $r=11.68$. 

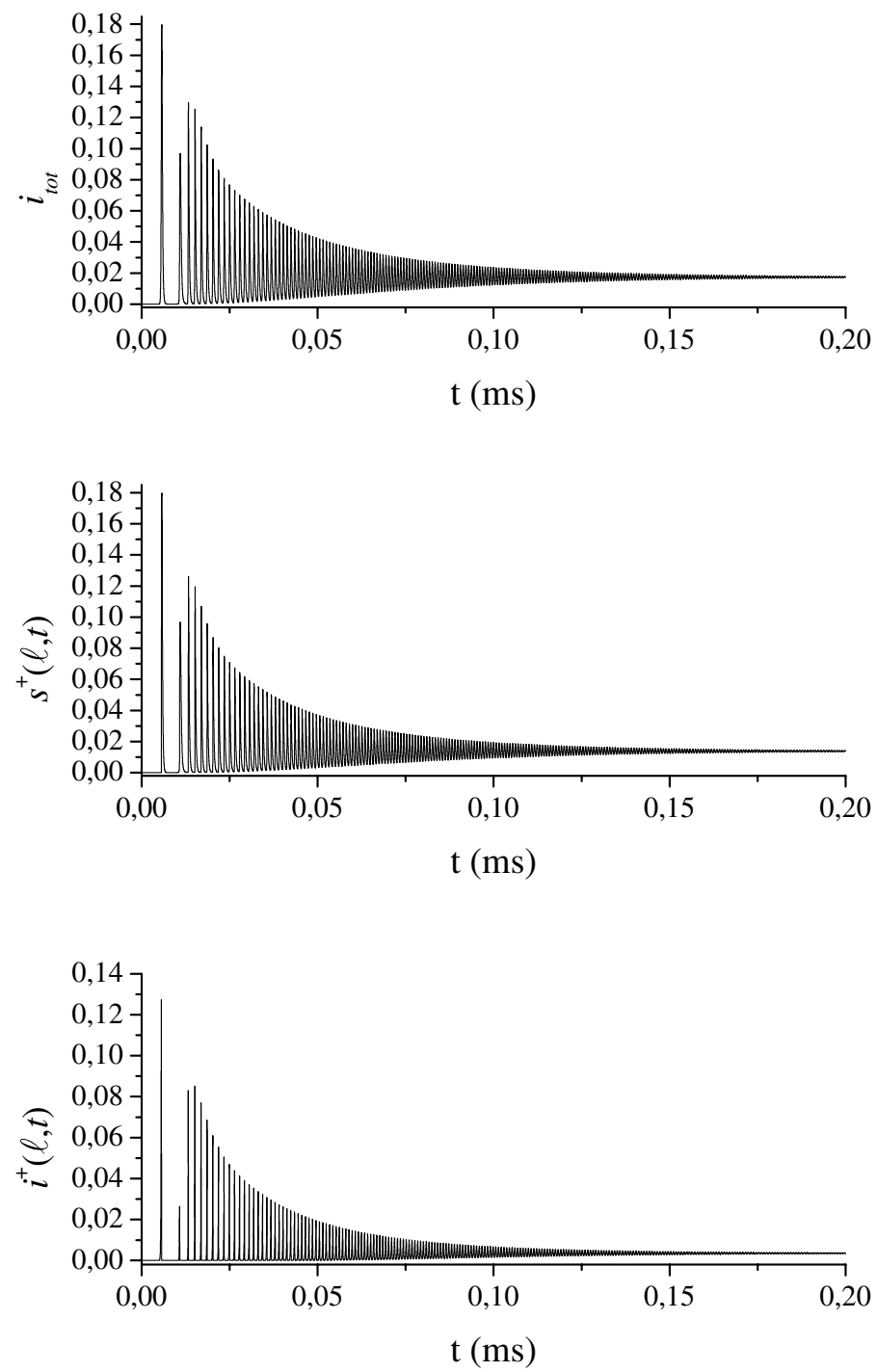

Fig. 4. Temporal evolution of the output laser $\left(i^{+}(\ell, t)\right)$ and stokes $\left(s^{+}(\ell, t)\right)$ intensities and of the total intensity $\left(i_{\text {tot }}\right)$ in a low-loss cavity. The pumping ratio is $r=6$.

Again, this is in agreement with experimental results reported in the Yb-doped fiber laser. ${ }^{17,18}$ Let us note that in this case, the suppression of the Brillouin interaction does not modify the stability of the laser. The only difference is an increase of the transient behaviour towards the continuous state.

\section{Conclusion}

In summary, we have proposed a simple theoretical approach for the modelling of a high-power fiber laser in the presence of Brillouin effect. The numerical results 
reported here are in rather good qualitative agreement with experimental data thus confirming the validity of our approach. Our model demonstrates, for the first time to our best knowledge, that Brillouin backscattering can induce a regular self-pulsing with a period large compared to the round-trip time in the cavity, for a fiber laser without any saturable absorption. As explained in the paper, some strong approximations have been done and there will be a lot of future works to account for other effects such as the finiteness of the phonon lifetime and optical Kerr nonlinearity. In spite of that, our model contains the minimal ingredients for a good description of the instabilities commonly occurring in high-power fiber laser.

\section{References}

1. R. G. Harrison, J. S. Uppal, A. Johnstone and J. V. Moloney, Evidence of chaotic stimulated Brillouin scattering in optical fibers, Phys. Rev. Lett. 65 (1990) 167-170.

2. F. Sanchez, Optique Nonlinéaire (Editions Ellipses, France, 1999).

3. A. Johnstone, W. Lu, J. S. Uppal and R. G. Harrison, Sustained and bursting oscillations in stimulated Brillouin scattering with external feedback in optical fiber, Opt. Com. 81 (1991) 222-224.

4. W. Lu and R. G. Harrison, Nonlinear dynamical and chaotic features in stimulated scattering phenomena, Europhys. Lett. 16 (1991) 655-660.

5. W. Lu, A. Johnstone and R. G. Harrison, Deterministic dynamics of stimulated scattering phenomena with external feedback, Phys. Rev. A 46 (1992) 4114-4122.

6. A. L. Gaeta and R. W. Boyd, Stochastic dynamics of stimulated Brillouin scattering in an optical fiber, Phys. Rev. A 44 (1991) 3205-3209.

7. M. Dämming G. Zinner, F. Mitschke and H. Welling, Stimulated Brilouin scattering in fibers with and without external feedback, Phys. Rev. A 48 (1993) 3301-3309.

8. R. G. Harrison, P. M Ripley and W. Lu, Observation and characterization of deterministic chaos in stimulated Brillouin scattering with weak feedback, Phys. Rev. A 49 (1994) R24-R27.

9. D. Yu, W. Lu and R. G. Harrison, Physical origin of dynamical stimulated Brillouin scattering in optical fibers with feedback, Phys. Rev. A 51 (1995) 669-674.

10. J. Botineau, C. Leycuras, C. Montes and E. Picholle, Stabilization of a stimulated Brillouin fiber ring laser by strong pump modulation, J. Opt. Soc. Am. B 6 (1989) 300-312.

11. C. Montes, D. Bahloul, I. Bongrand, J. Botineau, G. Cheval, A. Mamhoud, E. Picholle and A. Picozzi, Self-pulsing and dynamic bistability in cw-pumped Brillouin fiber ring lasers, J. Opt. Soc. Am. B 16 (1999) 932-951.

12. H. Li and K. Ogusu, Instability of stimulated Brillouin scattering in fiber ring resonator, Opt. Rev. 7 (2000) 303-308.

13. C. N. Pannell, P. St. J. Russell and T. P. Newson, Stimulated Brilouin scattering in optical fibers: The effects of optical amplification, J. Opt. Soc. Am. B 10 (1993) 684-690.

14. H. S. Kim, D.-K. Ko, G. Lim, B. H. Cha and J. Lee, The influence of laser gain on stimulated Brillouin scattering in an active medium, Opt. Com. 167 (1999) 165-170.

15. N. A. Brillant, Stimulated Brillouin scattering in a dual-clad fiber amplifier, J. Opt. Soc. Am. B 11 (2002) 2551-2557.

16. G. Kulcsar, Y. Jaouën, G. Canat, E. Olmedo and G. Debarge, Multiple-stokes stimulated Brillouin scattering generation in pulsed high-power double-cladding $\mathrm{Er}^{3+}-\mathrm{Yb}^{3+}$ codoped fiber amplifier, IEEE Phot. Techn. Lett. 15 (2003) 801-803. 
17. A. Hideur, T. Chartier, C. Özkul and F. Sanchez, Dynamics and stabilization of a high power side-pumped Yb-doped double-clad fiber laser, Opt. Com. 186 (2000) 311-317.

18. M. Salhi, A. Hideur, T. Chartier, M. Brunel, G. Martel, C. Özkul and F. Sanchez, Evidence of Brillouin scattering in an ytterbium-doped double-clad fiber laser, Opt. Lett. 27 (2002) 1294-1396.

19. B. Ortaç, A. Hideur, T. Chartier, M. Brunel, G. Martel, M. Salhi and F. Sanchez, Influence of cavity losses on stimulated Brillouin scattering in a self-pulsing sidepumped ytterbium-doped double-clad fiber laser, Opt. Com. 215 (2003) 389-395.

20. A. A. Fodiati, P. Mégret and M. Blondel, Dynamics of a self-Q-switched fiber laser with a Rayleigh-stimulated Brillouin scattering ring mirror, Opt. Lett. 29 (2004) 1078-1080.

21. M. Laroche, H. Gilles and S. Girard, Influence of stimulated Brillouin scattering on the temporal dynamics of an actively Q-switched double-clad fiber laser, Opt. Com. 281 (2008) 2243-2247.

22. H. Statz and G. De Mars, Multimode oscillations in solid-state masers, J. Appl. Phys. 35 (1964) 1377-1383.

23. F. Sanchez and G. Stephan, General analysis of instabilities in erbium-doped fiber lasers, Phys. Rev. E 53 (1996) 2110-2122.

24. F. Sanchez, M. Le Flohic, G. M. Stephan, P. Le Boudec and P. L. François, Quasiperiodic route to chaos in erbium-doped fiber laser, IEEE J. Quant. Electron. 31 (1995) 481-488.

25. G. P. Agrawal, Nonlinear Fiber Optics, 3rd edn. (Academic Press, New York, 2001). 